\title{
The application of the three-dimensional high-definition laparoscope in thyroid microcarcinoma
}

\author{
Yanming Dong, Shujia Peng, Haili Tang, Ping Yang, Lin Yang, Xiaojun Yang, Bo Qiu, Guoqiang Bao \\ Department of General Surgery, Tangdu Hospital, Air Force Medical University, Xi'an, China \\ Contributions: (I) Conception and design: Y Dong, G Bao; (II) Administrative support: All authors; (III) Provision of study materials or patients: Y \\ Dong, S Peng, H Tang, P Yang, L Yang, X Yang, B Qiu; (IV) Collection and assembly of data: Y Dong, S Peng, H Tang, P Yang, L Yang, X Yang, B \\ Qiu; (V) Data analysis and interpretation: Y Dong, G Bao; (VI) Manuscript writing: All authors; (VII) Final approval of manuscript: All authors. \\ Correspondence to: Guoqiang Bao, PhD. Department of General Surgery, Tangdu Hospital, Air Force Medical University, No. 1 Xinsi Road, Xi'an \\ 710038, China. Email: baoguoqiang_ln@163.com.
}

\begin{abstract}
Background: This study aimed to evaluate the feasibility and safety of the three-dimensional (3D) highdefinition (HD) laparoscope via a chest-breast approach in thyroid microcarcinoma.

Methods: In this retrospective study, ten patients with thyroid microcarcinoma who underwent laparoscopic thyroidectomy in the Department of General Surgery of Tangdu Hospital from May 2016 to October 2016 were included. Preoperative thyroid and neck ultrasound in these patients showed a thyroid nodule $\leq 1 \mathrm{~cm}$, and no significantly enlarged cervical lymph nodes were observed. The patients' thyroid function showed no subclinical hyperthyroidism. Three trocars were used via the chest and breast during the surgery. The main outcome measures included the operation time, intraoperative blood loss, postoperative hospital stay time, postoperative drainage volume, and the incidence of complications.
\end{abstract}

Results: The ten patients were successfully treated using a 3D HD laparoscope. The mean operation time was 70-160 minutes, the average intraoperative blood loss was 10-30 mL, the mean postoperative hospital stay was 4.5 days, and the mean postoperative drainage volume was $10-20 \mathrm{~mL}$. None of the patients needed to receive a traditional open thyroidectomy during the operation. No patient experienced hoarseness, numbness of limbs, or choking or coughing while drinking water.

Conclusions: The 3D endoscopic thyroidectomy operation via the chest-breast approach is a feasible and safe therapeutic method for the treatment of thyroid microcarcinoma.

Keywords: Three-dimensional high-definition laparoscope (3D HD laparoscope); thyroid microcarcinoma; chestbreast approach; intraoperative blood loss; postoperative drainage volume

Submitted Mar 16, 2020. Accepted for publication Nov 16, 2020.

doi: $10.21037 /$ tcr-20-1525

View this article at: http://dx.doi.org/10.21037/tcr-20-1525

\section{Introduction}

With the increasing health awareness of the world's population, thyroid nodules are often found in annual general physical examinations (1-3). Many patients with thyroid nodules (benign or malignant) require surgical treatment. Conventional thyroidectomy involves making a transverse incision about 6-8 cm long in the neck. However, thyroid disorders are more common in young women, who often feel uncomfortable and cosmetically unacceptable with the scars caused by the incision (4-6). Before the endoscopic thyroidectomy was first introduced by Hüscher et al. (7) in 1997, endoscopic thyroidectomy operations were usually performed on patients with benign thyroid nodules. As surgical knowledge and experience accumulated, the application of the endoscopic thyroidectomy was extended to patients with thyroid carcinoma at an early stage, particularly thyroid microcarcinoma. With the rapid expansion of endoscopic equipment, surgery methods using 
two-dimensional (2D) techniques were promptly applied in various medical fields.

Recently, three-dimensional (3D) high-definition (HD) laparoscope technology has progressively been applied and has become popular in surgical fields. Many researchers have also reported the feasibility and safety of the $2 \mathrm{D}$ endoscopic technique thyroidectomy when compared to the conventional open thyroidectomy (8). Therefore, in this study, we aimed to evaluate the feasibility and safety of the 3D HD laparoscope via the chest-breast approach for the treatment of thyroid microcarcinoma. We present the following article in accordance with the STROBE reporting checklist (available at http://dx.doi.org/10.21037/tcr-201525).

\section{Methods}

\section{Subjects}

In this retrospective study, ten patients with thyroid microcarcinoma who underwent laparoscopic thyroidectomy in the Department of General Surgery of Tangdu Hospital of the Fourth Military Medical University from May 2016 to October 2016 were included as subjects. Preoperative thyroid and neck ultrasound in these patients showed a thyroid nodule $\leq 1 \mathrm{~cm}$, and no significantly enlarged cervical lymph nodes were observed. The preoperative examination results of patients' routine blood, liver and kidney function, coagulation function, electrocardiogram, chest X-ray, lung function, etc. were normal. The patients' thyroid function [T3, T4, thyroidstimulating hormone (TSH)] showed no subclinical hyperthyroidism. One surgeon (Dr. G Bao) performed all the procedures. The study was conducted in accordance with the Declaration of Helsinki (as revised in 2013). The study was approved by institutional/regional/national ethics/committee/ethics board of Tangdu Hospital, Air Force Medical University and informed consent was taken from all the patients.

\section{The inclusion and exclusion criteria}

The inclusion criteria for this study were as follows: (I) patients $>18$ years; (II) thyroid nodules were diagnosed as malignancy by the preoperative ultrasound (US)-guided fine-needle aspiration and cytology (FNAC) or suspicious malignancy by preoperative neck US, neck CT, thyroid microcarcinoma smaller than $10 \mathrm{~mm}$ in diameter, and no evidence of central or lateral lymph node metastasis; (III) patients and their family members were worried about scarring and accepted the possibility that an open thyroidectomy may need to occur during the operation. The exclusion criteria were as follows: (I) patients with hyperthyroidism and thyroid surgery; (II) patients with severe infections; (III) patients with severe coagulation dysfunction.

\section{Surgical technique}

All patients were prepared for the endoscopic thyroidectomy under general anesthesia. After the patient was placed in a supine astride position, a pillow was placed beneath the patient's shoulder to extend the head and neck. The operator and scope assistant stood between the legs and on the right side of the patient. The first assistant stood on the left side of the patient, and the monitors were placed on the head side of the patient (Figure 1). In order to facilitate the dissection and reduce bleeding, approximately $500 \mathrm{~mL}$ of normal saline solution (including $1 \mathrm{~mL}$ epinephrine) was injected into the subcutaneous layer of the anterior chest. A $5 \mathrm{~mm}$ incision was made on the upper edge of the areola on the right side, a $10 \mathrm{~mm}$ incision was made on the upper edge of the areola on the left side, and a $10 \mathrm{~mm}$ incision was made in the right parasternal region (Figure 2). Initially, the flaps were dissected bluntly with a glass rod bar. After blunt dissection, the trocars were inserted through each incision. Carbon dioxide gas was injected with a pressure of 6-8 mmHg. A 3D HD laparoscope (Olympus, Tokyo, Japan) was inserted through the trocar in the right parasternal region. A retractor was stuck on the lateral side of the neck and drew back the sternocleidomastoid muscles to create adequate working space. The working space was extended from the anterior chest to the thyroid cartilage level and laterally to the medial edge of each of the sternocleidomastoid muscles. After the flap extension was completed, a midline division was made between the strap muscle from the exposed and dissected thyroid gland or isthmus of the thyroid gland according to the principles of a thyroidectomy (thyroid middle vein, isthmus, inferolateral thyroid vessel, and thyroid upside vessel). The isthmus of the thyroid gland was divided using ultrasonic shears. After careful dissection of the inferolateral aspects of the thyroid gland, the recurrent laryngeal nerve (RLN) and the inferior parathyroid gland were identified. The inferior thyroidal artery and the middle thyroidal vein were identified and divided. The superior pole of the gland was dissected, and 


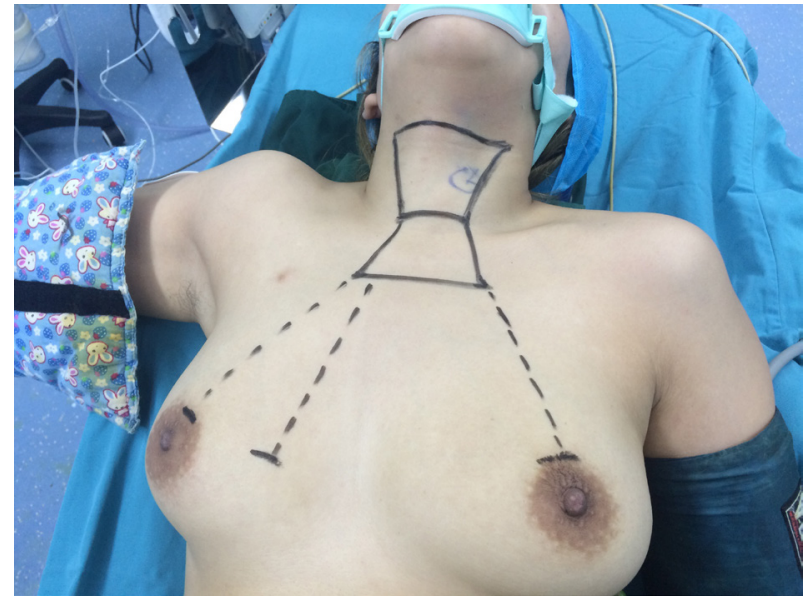

Figure 1 Three trocars position in the chest. Trocar A is aided hole, trocar B is endoscopic hole, trocar C is main operating hole. Cervico-thoracic line region shows extent of free skin flaps during operation.
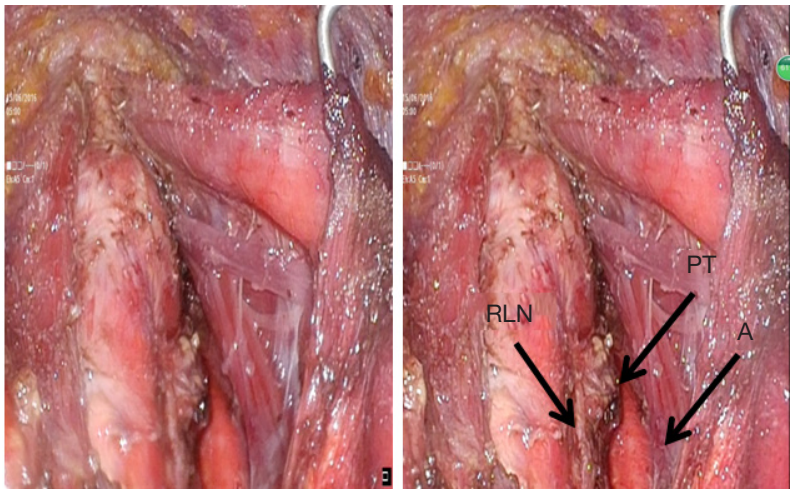

Figure 2 The operation picture is operating field image by $3 \mathrm{D}$ HD. RLN, recurrent laryngeal nerve; PT, parathyroid; A, common carotid artery.

the superior thyroid vessel was divided with ultrasonic shears. After the laryngeal nerve was wholly exposed, the thyroid gland was separated from the trachea.

After being placed in an endo-bag, the specimen was pulled out through the endoscope port site. A frozen section of the specimen was examined intraoperatively for pathological confirmation. After thyroid microcarcinoma was diagnosed, the central lymph node dissection was performed and sent to the pathology department for a quick examination. The central lymph node was examined for metastatic cancer by ultrasound or cervical CT. The cavity was cleaned with a saline solution before meticulous hemostasis was performed. The strap muscles were approximated with an absorbable suture, and a 100-cc drainage tube was left in the operative bed through the endoscope left port site.

\section{Main outcome measures}

The main outcome measures included the operation time, intraoperative blood loss, postoperative hospital stay time, postoperative drainage volume, and the incidence of complications.

\section{Statistical analysis}

SPSS 20.0 (IBM, Chicago, USA) was used for statistical analysis. Continuous variables were expressed as mean \pm SD. Discontinuous variables were expressed as a percentage (\%). $\mathrm{P}<0.05$ was considered statistically significant.

\section{Results}

\section{Baseline data}

A total of ten patients were included in this study, comprising two males and eight females. These patients were diagnosed with thyroid microcarcinoma and underwent a 3D endoscopic thyroidectomy via the chestbreast approach with or without central lymph node dissection. The average age of these patients was 35.5 years (range, 23-48 years) (Table 1).

\section{The main outcome measures}

All ten patients were successfully treated with endoscopic thyroidectomy, and none of the patients needed to receive a traditional open thyroidectomy during the operation. The mean hospital stay was $5.0 \pm 1.5$ days. The total operation time was $95 \pm 25$ minutes (including the intraoperative freezing pathological examination time). Intraoperative bleeding was $20 \pm 10 \mathrm{~mL}$ (Table 1). The time of removing the drainage was $3.0 \pm 1.5$ days. Trocar channel uncontrolled bleeding occurred in one patient and was successfully controlled by the local strapping of the operated chest wall with a crepe bandage. Subcutaneous emphysema of the cervical lacuna presented in one patient, and it had vanished from sight on the third day after the operation, which did not cause discomfort to the patient. Transient 
Table 1 Clinical data and surgical outcomes of the patients

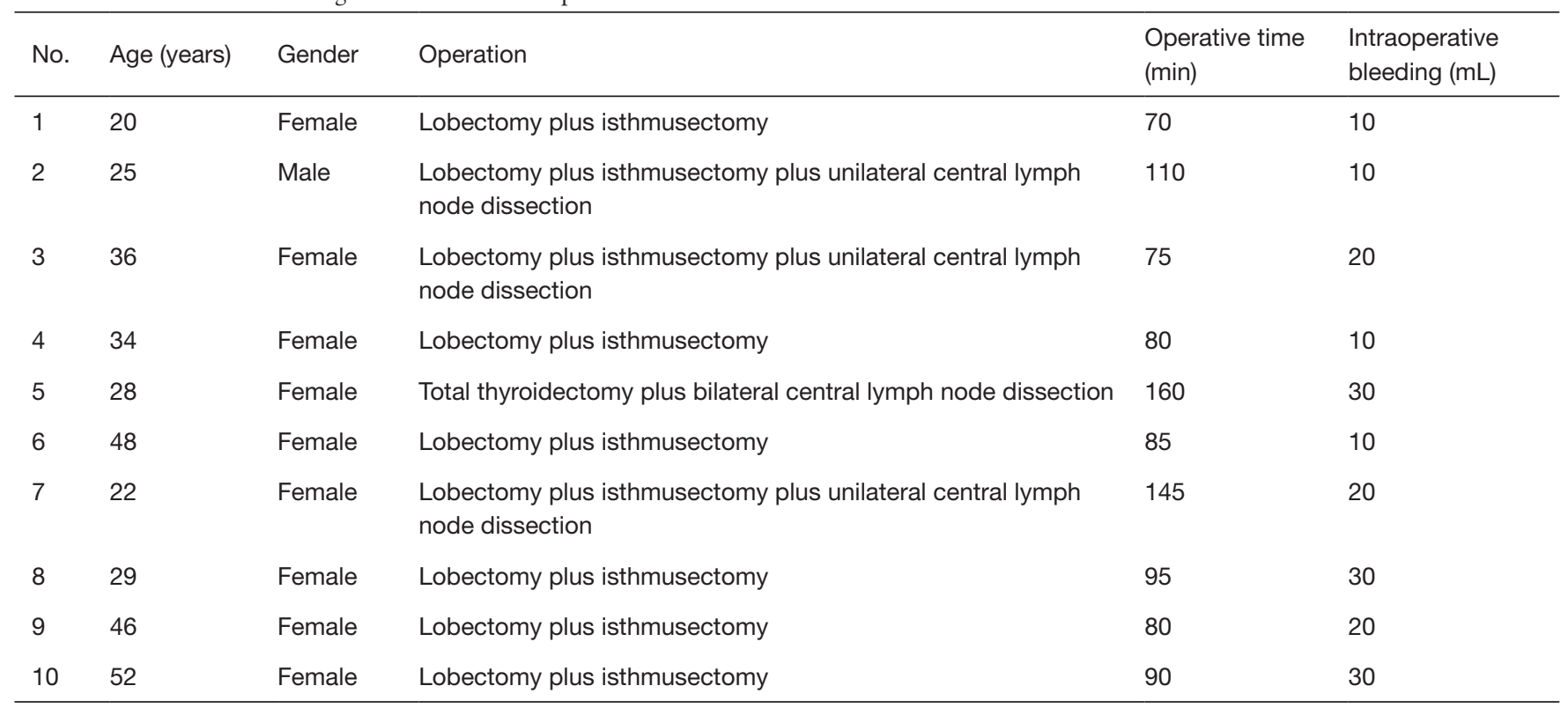

vocal cord paresis occurred in two patients. On the first day after the operation, one patient experienced acroaesthesia and muscular spasm non-hyperspasmia. These symptoms were relieved after giving the patient a calcium supplement. No patient experienced hoarseness, numbness of limbs, or choking or coughing while drinking water.

\section{Discussion}

Most patients with thyroid microcarcinoma are young women. The minimally invasive endoscopic thyroidectomy emerged and showed well-accepted results with an improved cosmetic outcome, accelerated healing, and more comfort and acceptability in patients. Our study aimed to evaluate the feasibility and safety of the 3D HD laparoscope via the chest-breast approach for the treatment of thyroid microcarcinoma.

In this study, ten patients were treated successfully with a 3D HD laparoscope. The results showed that the mean operation time was $70-160$ minutes, the average intraoperative blood loss was $10-30 \mathrm{~mL}$, the mean postoperative hospital stay was 4.5 days, and the mean volume of the drainage after the surgery was $10-20 \mathrm{~mL}$.

The main advantages of the 3D laparoscope compared with the 2D laparoscope are reflected by the feeling of depth in the surgical field and the stronger spatial location expression (Figure 2). In particular, it performs outstandingly in terms of the exposure and overall protection of the RLN and the in-situ retention of the bilateral parathyroid during the thyroid operation. Generally, the difficulty during thyroidectomy is the treatment of the RLN throat entrance. The 3D laparoscope with HD amplification and stereoscopic vision can clear the exposure and spatial relation of the nerve branches and the small nourish vessels at this point, which avoids the hemorrhage of the small vessels and the deputy injury of the RLN and parathyroid. The 3D laparoscope has a more accurate command of the incisional margin and distance and can complete a detailed operation. For example, the stereoscopic vision effect is more obvious when there are a laparoscopic anatomic separation and a knot-tying suture. It is a great help in creating knot-tying sutures and the knot of the needle-holder. Therefore, it is easier and has a low error rate for surgeons during thyroid surgery.

In the small sample data, the operation time with a $3 \mathrm{D}$ laparoscope was much the same as the time with a $2 \mathrm{D}$ laparoscope using the same surgery methods, which had the same conclusion as the research of cholecystectomy with 3D laparoscopes by HANNA (8). However, the BILGEN (9) group contrasted the time of the $3 \mathrm{D}$ system and the most popular 2D system using similar methods as the HANNA group, which had the completely contrary conclusion that 
a 3D laparoscope can effectively shorten surgery time and surgeons would accept it more easily. The study of the PEITGEN (10) group also showed that the 3D image could significantly shorten surgery time regardless of whether surgeons had rich laparoscope operation experience or not. The experiment completed by BHAYANI (11) demonstrated that laparoscopy beginners performed better when operating with stereoscopic images than with the traditional 2D images. The operation with the stereoscopic image took a shorter time and was accepted by testers more easily than the 2D images (12-14). However, in this paper, the amount of bleeding, postoperative suction drainage, and hospital stay after 3D laparoscopic surgery had no obvious difference from 2D laparoscopic surgery. However, a 3D laparoscope may have several limitations. A slight tremor of the hand holding the laparoscope or the fast and minor lens adjustment causes the image to shake because of the HD amplification effect of the 3D laparoscope, leading to the operators' visual discomfort or dizziness (15-17). Unlike the 2D laparoscope, the $3 \mathrm{D}$ laparoscope lacks a lens with a 30-degree rotation, and the finite angle bending at the end of the lens causes a surgery-blind area that increases the operation's difficulty, the surgery risk, and the incidence of postoperative complications (18-20). In short, with the continuous improvement of the laparoscope, minimally invasive surgery plays an important role in many medical fields. Thus, the application of the $3 \mathrm{D}$ laparoscope in thyroid surgery is becoming increasingly frequent.

There were some limitations in our study. (I) This trial was an observational trial and not a randomized controlled trial, and no control group was designed in this study. (II) This study was only a single-center trial, and the sample size was small. Multi-center studies with large samples are still needed to confirm the results of our study. (III) The clinical follow-up was short, and it was necessary to observe the long-term clinical prognosis. (IV) The difference between 3D laparoscope and 2D laparoscope for thyroid microcarcinoma remains unknown and should be further researched.

\section{Conclusions}

In this study, we observed the safety and feasibility of $3 \mathrm{D}$ endoscopic thyroidectomy operations via the chest-breast approach to treat thyroid microcarcinoma and found that the operation time and postoperative hospital stay time were relatively short, and the intraoperative blood loss and postoperative drainage volume were small. Most importantly, no patient experienced severe complications such as hoarseness, numbness of limbs, or choking or coughing while drinking water. In conclusion, the $3 \mathrm{D}$ endoscopic thyroidectomy operation via the chest-breast approach may be a preferable therapeutic method for patients with thyroid microcarcinoma. We will conduct research with large samples in the future for a deeper exploration of this technology.

\section{Acknowledgments}

We are particularly grateful to all the people who have given us help on our article.

Funding: This work was supported by the National Natural Science Foundation of China (grant no. 81572916).

\section{Footnote}

Reporting Checklist: The authors have completed the STROBE reporting checklist. Available at http://dx.doi. org/10.21037/tcr-20-1525

Data Sharing Statement: Available at http://dx.doi. org/10.21037/tcr-20-1525

Conflicts of Interest: All authors have completed the ICMJE uniform disclosure form (available at http://dx.doi. org/10.21037/tcr-20-1525). The authors have no conflicts of interest to declare.

Ethical Statement: The authors are accountable for all aspects of the work in ensuring that questions related to the accuracy or integrity of any part of the work are appropriately investigated and resolved. The study was conducted in accordance with the Declaration of Helsinki (as revised in 2013). The study was approved by institutional/ regional/national ethics/committee/ethics board of Tangdu Hospital, Air Force Medical University and informed consent was taken from all the patients.

Open Access Statement: This is an Open Access article distributed in accordance with the Creative Commons Attribution-NonCommercial-NoDerivs 4.0 International License (CC BY-NC-ND 4.0), which permits the noncommercial replication and distribution of the article with the strict proviso that no changes or edits are made and the original work is properly cited (including links to both the formal publication through the relevant DOI and the license). 
See: https://creativecommons.org/licenses/by-nc-nd/4.0/.

\section{References}

1. Dean DS, Gharib H. Epidemiology of Thyroid Nodules. Best Pract Res Clin Endocrinol Metab 2008;22:901-11.

2. Durante C, Grani G, Lamartina L, et al. The Diagnosis and Management of Thyroid Nodules: A Review. JAMA 2018;319:914-24.

3. Li MH, Liu JT. Screening of benign and malignant thyroid nodules in 5196 physical examination population. Zhonghua Zhong Liu Za Zhi 2018;40:151-4.

4. Zhang D, Cai K, Wang G, et al. Trimester-specific reference ranges for thyroid hormones in pregnant women. Medicine (Baltimore) 2019;98:e14245.

5. Korevaar TIM, Mínguez-Alarcón L, Messerlian C, et al. The association of thyroid function and autoimmunity with ovarian reserve in women seeking infertility care. Thyroid 2018;28:1349-58.

6. Kannan S, Mahadevan S, Sigamani A. A Systematic Review on Normative Values of Trimester-specific Thyroid Function Tests in Indian Women. Indian J Endocrinol Metab 2018;22:7-12.

7. Hüscher CS, Chiodini S, Napolitano C, et al. Endoscopic Right thyroid lobectomy. Surg Endosc 1997;11:877.

8. Hanna GB, Shimi SM, Cuschieri A. Randomised study of influence of two-dimensional versus three-dimensional imaging on performance of laparoscopic cholecystectomy. Lancet 1998;351:248-51.

9. Bilgen K, Ustün M, Karakahya M, et al. Comparison of 3D imaging and 2D imaging for performance time of laparoscopic cholecystectomy. Surg Laparosc Endosc Percutan Tech 2013;23:180-3.

10. Peitgen K, Walz MV, Walz MV, et al. A prospective randomized experimental evaluation of three-dimensional imaging in laparoscopy. Gastrointest Endosc 1996;44:262-

Cite this article as: Dong Y, Peng S, Tang H, Yang P, Yang L, Yang X, Qiu B, Bao G. The application of the three-dimensional high-definition laparoscope in thyroid microcarcinoma. Transl Cancer Res 2021;10(2):977-982. doi: 10.21037/tcr-20-1525
7.

11. Bhayani SB, Andriole GL. Three-Dimensional (3D) Vision: Does It Improve Laparoscopic Skills? An Assessment of a 3D Head-Mounted Visualization System. Rev Urol 2005;7:211-4.

12. Zhao D, Huang Z, Zou Z. Research progress of threedimensional laparoscope system. Nan Fang Yi Ke Da Xue Xue Bao 2014;34:594-6, 1 p following 596.

13. Ai Y, Pan B, Fu Y, et al. Design of a novel robotic system for minimally invasive surgery. Ind Rob 2017;44:288-98.

14. Manning TG, Papa N, Perera M, et al. Laparoscopic lens fogging: solving a common surgical problem in standard and robotic laparoscopes via a scientific model. Surg Endosc 2018;32:1600-6.

15. Panday BC, Malhotra S, Sood J. Laparoscope surgical Instrument usage to manage an extremely difficult airway. J Clin Anesth 2018;45:62.

16. McDougall EM, Soble JJ, Wolf JS Jr, et al. Comparison of Three-Dimensional and Two-Dimensional Laparoscopic Video Systems. J Endourol 1996;10:371-4.

17. Yu W, Liu T, Li C, et al. Comparative study of 3D and 2D laparoscopic surgery for gastrointestinal tumors. Zhonghua Wei Chang Wai Ke Za Zhi 2015;30:297-9.

18. Chen H, Yu J, Huang Z, et al. Application of threedimensional high-definition laparoscope in laparoscopic radical resection of gastric cancer. Nan Fang Yi Ke Da Xue Xue Bao 2014;34:588-90.

19. Watanabe $Y$, Sato $M$, Abe $Y$, et al. Three-dimensional arterial computed tomography and laparoscope-assisted splenectomy as a minimally invasive examination and treatment of splenic aneurysms. J Laparoendosc Adv Surg Tech A 1997;7:183-6.

20. Schwab K, Smith R, Brown V, et al. Evolution of stereoscopic imaging in surgery and recent advances. World J Gastrointest Endosc 2017;9:368-77. 